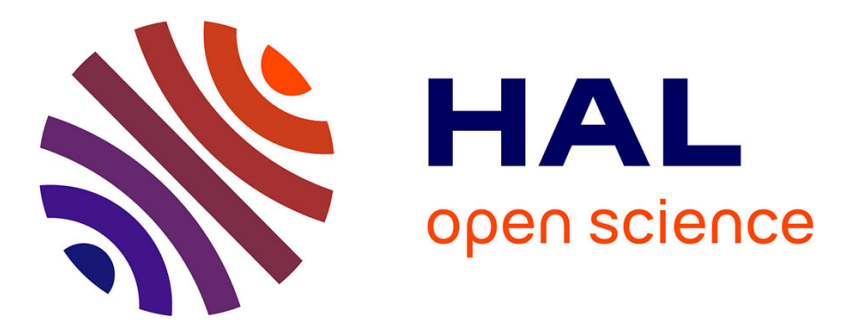

\title{
Temperature dependence of non-radiative relaxation probabilities of Cr3+ in BIGaZYT fluoride glass measured by a pulsed photoacoustic technique
}

\author{
A. Mendioroz, J. Fernández, R. Balda, C. Folcia
}

\section{To cite this version:}

A. Mendioroz, J. Fernández, R. Balda, C. Folcia. Temperature dependence of non-radiative relaxation probabilities of $\mathrm{Cr} 3+$ in BIGaZYT fluoride glass measured by a pulsed photoacoustic technique. Journal de Physique IV Proceedings, 1994, 04 (C7), pp.C7-413-C7-416. 10.1051/jp4:1994797 . jpa00253328

\section{HAL Id: jpa-00253328 \\ https://hal.science/jpa-00253328}

Submitted on 1 Jan 1994

HAL is a multi-disciplinary open access archive for the deposit and dissemination of scientific research documents, whether they are published or not. The documents may come from teaching and research institutions in France or abroad, or from public or private research centers.
L'archive ouverte pluridisciplinaire HAL, est destinée au dépôt et à la diffusion de documents scientifiques de niveau recherche, publiés ou non, émanant des établissements d'enseignement et de recherche français ou étrangers, des laboratoires publics ou privés. 


\title{
Temperature dependence of non-radiative relaxation probabilities of $\mathrm{Cr}^{3+}$ in BIGaZYT fluoride glass measured by a pulsed photoacoustic technique
}

\author{
A. Mendioroz, J. Fernández, R. Balda and C.L. Folcia* \\ ETSII y Telecom, Dpto. Física Aplicada I, Universidad del País Vasco, Alda, Urquijo s/n, 48013 Bilbao, \\ Spain \\ * Fac. Ciencias, Dpto. Física Materia Condensada, Universidad del País Vasco, Apartado 644, Bilbao, \\ Spain
}

\begin{abstract}
The temperature dependence of the non-radiative relaxation probabilities of $\mathrm{Cr}^{3+}$ in fluoride BIGaZYT glass have been obtained by pulsed photoacoustic technique. The results are compared with nonradiative probabilities obtained from luminescence measurements.
\end{abstract}

\section{INTRODUCTION}

The theory of the pulsed photoacoustic signal (PAS) generation has been widely studied in liquids and gases $^{(1)}$. Solid samples have been essentially treated in the case of opaque materials (metals and semiconductors). However, the photoacoustic pulsed technique is also a useful tool to investigate the nonradiative deexcitation probabilities of optically active ions in solid hosts.

In this work we present a theoretical approach to the photoacoustic waveforms generated by a thermal expansion mechanism in pulsed regime, obtained for optically thin and thermally thick isotropic solid samples. In particular, the attention is focused on the PAS generation due to non-radiative deexcitation of $\mathrm{Cr}^{3+}$ in BIGaZYT fluoride glass. From the theoretical results and the measured photoacoustic waveforms as a function of temperature, the behaviour of the non-radiative deexcitation probability of $\mathrm{Cr}^{3+}$ for the ${ }^{4} \mathrm{~T}_{2} \rightarrow{ }^{4} \mathrm{~A}_{2}$ transition has been found in the $77-300 \mathrm{~K}$ temperature range.

\section{THEORY}

The pressure exerted by the sample on the transducer has been obtained by assuming a two level system microscopic scheme for the relaxation of the $\mathrm{Cr}^{3+}$ ions. The basic equations governing the PAS generation were derived from thermoelastic considerations ${ }^{(2)}$. Neglecting the thermal diffusivity, the scalar potential $\Psi$ associated to the longitudinal component of the displacement verifies the following differential equation

$$
\nabla^{2} \psi-\frac{1}{c^{2}{ }_{1}} \frac{\partial^{2} \psi}{\partial t^{2}}=\frac{3 K \beta}{3 K+4 \mu} \frac{1}{\rho c_{p}} \int_{0}^{t} H(t) d t
$$

where $c_{1}$ is the velocity of the longitudinal wave, $\beta$ the thermal expansion coefficient under constant pressure, $K$ and $\mu$ are the bulk and shear moduli, $\rho$ is the density, $c_{p}$ the specific heat at constant pressure, and $H(t)$ the heat released per unit time and unit volume, obtained from the deexcitation scheme. In isotropic elastic solids, the potential $\Psi$ is related to the pressure on an elementary surface, normal to $\mathbf{n}$ by:

$$
\mathbf{p}=(\lambda+\mu)(\operatorname{div}(\operatorname{grad} \Psi)) \mathbf{n}+\mu \operatorname{grad}(\mathbf{n} \cdot(\operatorname{grad} \Psi))+\mu \operatorname{rot}((\operatorname{grad} \Psi) \times \mathbf{n})
$$


where $\lambda$ and $\mu$ are the Lamé constants. For cylindrical symmetry and neglecting the contribution of transverse components of the displacement (assuming an infinite medium) the radial component of pressure (normal to the surface of the sample), takes the form:

$$
\mathrm{p}_{\mathrm{r}}^{\mathrm{r}}=(\lambda+2 \mu) \frac{\partial^{2} \psi}{\partial \mathrm{r}^{2}}+(\lambda+\mu) \frac{1}{\mathrm{r}} \frac{\partial \psi}{\partial \mathrm{r}}
$$

The source function in equation (1) is proportional to the integrate of the heat released in the sample per unit time per unit volume. Its analytical form can be deduced from a simple microscopic scheme of the interaction between ions and photons. $\mathrm{Cr}^{3+}$ ion occupies low field sites in these matrices and behaves as a two level system, shown schematically in the next figure:

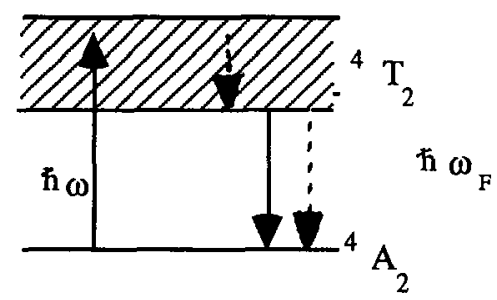

Figure1: Level scheme for $\mathrm{Cr}^{3+}$ in low field sites

Energy from the pumping beam (a pulsed laser with pulse duration of $\tau_{\mathrm{L}} \approx 10 \mathrm{~ns}$ ) excites the electrons from the ${ }^{4} A_{2}$ to the ${ }^{4} T_{2}$ energy band. Electronic relaxation to the lower excited state level takes place with a time constant of the order of picoseconds $\left(\ll \tau_{\mathrm{L}}\right)$, and the lifetime of electrons in the lower excited state level is of the order of microseconds ( $» \tau_{\mathrm{L}}$ ). The great difference between the laser pulse duration and the lifetime of electrons in the pumping level allows us to neglect the shortest time and assume that the filling of the lowest excited state is governed by the laser time profile.

According to this scheme, the heat generated per unit time and unit volume in the sample can be written as follows:

$$
H=H_{1}+H_{2}=\frac{\alpha I}{\hbar \omega}\left(\hbar \omega-\hbar \omega_{F}\right)+\frac{N_{2}}{\tau}(1-\eta) \hbar \omega_{F}
$$

where $\alpha$ is the absorption coefficient, $I$ is the intensity of the laser beam, $\hbar \omega$ and $\hbar \omega_{F}$ are the pumping and fluorescence photon energy, $\mathrm{N}_{2}$ is the number of ions per unit volume in the excited state lower level, $\tau$ is the lifetime of the electron in this level, and $\eta$ is the quantum efficiency of fluorescence. As is evident, $\mathrm{H}(\mathrm{r}, \mathrm{t})$ can be written in two terms, a first one $\mathrm{H}_{1}$ corresponding to the fast nonradiative decay to the bottom of the absorption band, and a second one $\mathrm{H}_{2}$ which describes the slow de-excitation from that level to the ground state. Introducing $\mathrm{H}_{1}(\mathrm{r}, \mathrm{t})$ and $\mathrm{H}_{2}(\mathrm{r}, \mathrm{t})$ into the right-hand side of equation for $(1)$, one gets two wave equations which correspond to potential functions associated to the fast and slow de-excitation processes. The dependence of $\mathrm{N}_{2}$ with time arises from the solution of the rate equation

$$
\frac{\mathrm{dN}_{2}}{\mathrm{dt}}=\frac{\alpha \mathrm{I}}{\hbar \omega}-\frac{\mathrm{N}_{2}}{\tau}
$$

The theoretical photoacoustic waveforms generated by the fast relaxation inside the ${ }^{4} \mathrm{~T}_{2}$ band and by the deexcitation from the relaxed excited state to the ${ }^{4} \mathrm{~A}_{2}$ ground state can be obtained by solving equation (1) by the Green method, with the Green function(3),

$$
G\left(\mathbf{r}, \mathbf{r}^{\prime}, t, t^{\prime}\right)=\frac{1}{\sqrt{v^{2}\left(t-t^{\prime}\right)^{2}-\left|r-r^{\prime}\right|^{2}}} \quad \text { for } t \geq t^{\prime}+\frac{|\mathbf{r}-\mathbf{r}|}{v}
$$


Figure 2 shows the results for typical constant values, asymmetric temporal laser profile of 9 ns width, lifetime of the excited state $\tau$ of $100 \mathrm{~ns}$, and a zero radius laser beam. As can be seen, at early times the pulse generated by deexcitation to the relaxed excited state (circles) does not essentially differ from the total response, which is the sum of both pulses (solid line). Moreover, the photoacoustic signal generated by the deexcitation from the relaxed excited state to the ground state (crosses) is proportional to the non-radiative deexcitation probability.

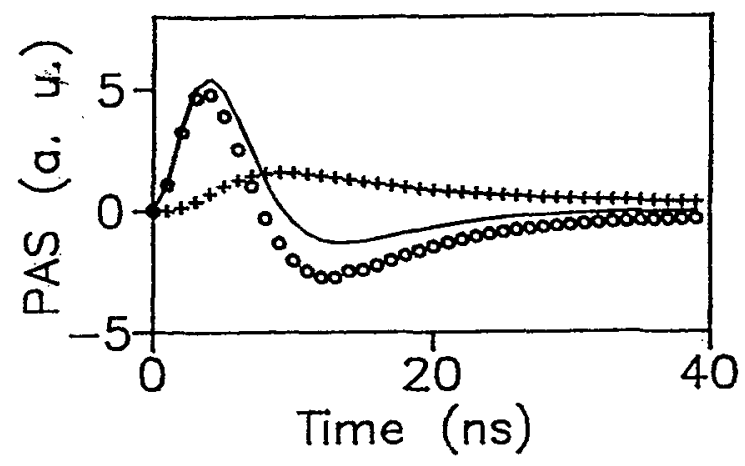

Figure 2: PAS generated by the fast (circles) and slow (100 ns) deexcitations (crosses) and sum of both (solid line).

\section{EXPERIMENTAL}

Experimental measurements were carried out in a BIGaZYT: $\mathrm{Cr}^{3+}(0.5 \mathrm{~mol} \%)$ fluoride glass sample with a pulsed frequency doubled YAG:Nd pumped tunable dye laser of $9 \mathrm{~ns}$ pulse width and $0.08 \mathrm{~cm}^{-1}$ linewidth, tuned at $630 \mathrm{~nm}$. A fraction of the incident beam was split to fall upon an area of the sample covered with black paint. The signal from this beam was used to normalize the temperature response of the system that was varied from 77 to $300 \mathrm{~K}$. A photoacoustic cell was constructed to operate inside an Oxford continuous flow cryostat. The signal was detected by a $25 \mu \mathrm{m}$ thick PVDF piezoelectric film, amplified by a 115 wideband EG\&G-PAR pre-amplifier and finally monitored on a Tektronix 2440 digital scope connected to a computer. Figure 3 shows a typical experimental waveform at room temperature.

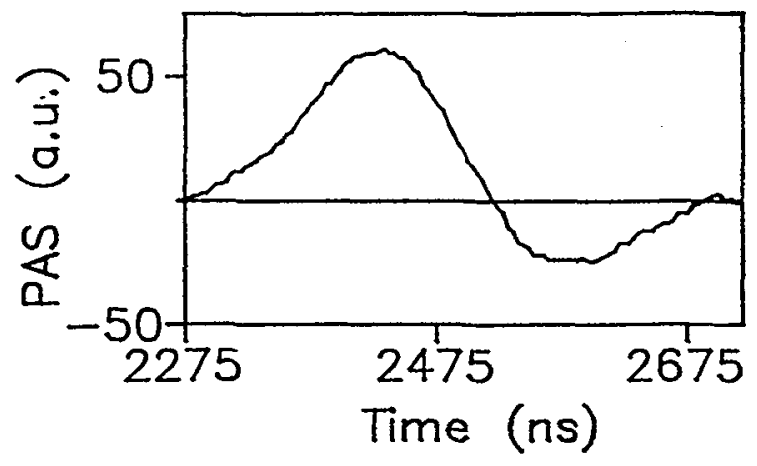

Figure 3: Typical experimental waveform at room temperature. 


\section{RESULTS AND DISCUSSION}

Next we present the results obtained for the pressure corresponding to the first deexcitation and to the second one for different lifetimes of the metastable level (figure 4).

Inspection of the curves shows that the photoacoustic signal decreases in amplitude and increases in width when the lifetime of electrons in the metastable level increases. This is in qualitative agreement with the theoretical results obtained by Kuo et al.(4) for liquid samples.

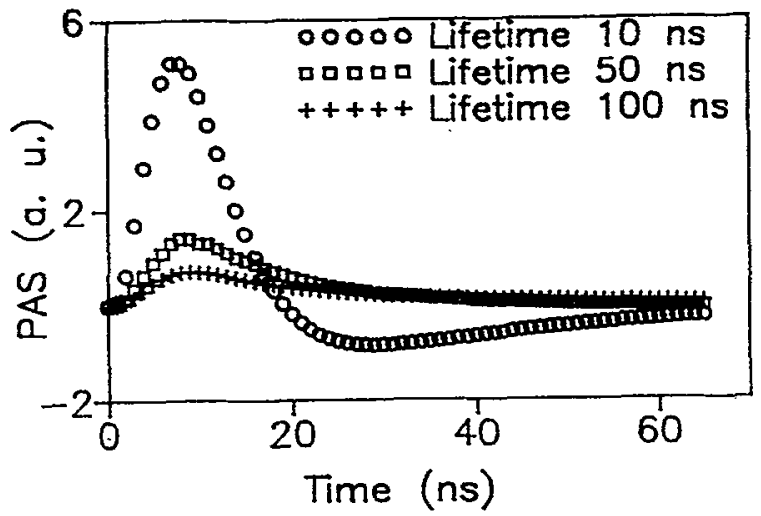

Figure 4: PAS generated by the slow deexcitation for three different lifetimes.

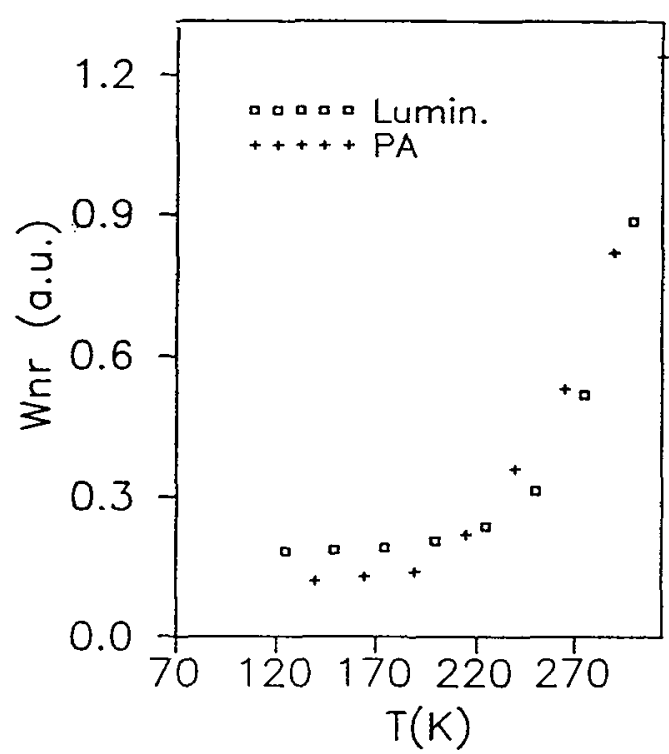

Figure 5: Non-radiative relaxation probabilities obtained from photoacoustic and luminescence measurements.

From the results shown in figure 2 the temperature dependence of the non-radiative relaxation probability can be obtained. Assuming a given value for the $\mathrm{Cr}^{3+}$ quantum efficiency at liquid Nitrogen temperature (LNT) and accepting there is no variation with temperature of the deexcitation inside the ${ }^{4} \mathrm{~T}_{2}$ band, the scaled difference between the photoacoustic signal at a certain temperature and the signal at LNT gives the relative contribution of the heat released from the metastable level at that temperature. As theoretical results point out, the substraction must be carried out after scaling both curves at early times. The amplitude of the difference is proportional to the non-radiative deexcitation probability $\mathrm{W}_{\mathrm{nr}}$. Figure 5 shows the experimental behaviour of $\mathrm{W}_{\mathrm{nr}}$ with temperature compared to the values obtained by luminescence spectroscopy [5].

\section{References}

[1] Diebold G.J. et al., Phys. Rev. Letters 67 (1991) 3384, and references therein.

[2] Liu G., Appl. Optics 21 (1982) 955.

[3] Morse P. M. and Feshbach H., Methods of theoretical Physics Vol I. (McGraw-Hill, New York, 1953)

[4] Kuo Ch.-Y., Vieira M. M. F., and Patel C. K. N., J. Appl. Phys. 55 (1984) 333-3336.

[5] Illarramendi M. A., Fernández J., Balda R., and Adam J. L., J. of Lumin. 47 (1991) 207. 